\title{
Prospective randomised controlled trial of methods of call and recall for cervical cytology screening
}

\author{
Mary Pierce, Simon Lundy, Aileen Palanisamy, Sue Winning, Jackie King
}

\begin{abstract}
Objective-To discover whether systematic methods of call and recall are more effective than a non-systematic method and to see which of the two systematic methods was more effective.
\end{abstract}

Design-Prospective randomised controlled trial over a year.

Setting-One group general practice.

Patients-416 Women over 35 eligible for a smear test who had never had a cervical smear test or in whom a smear test was overdue (previous test more than five years before).

Interventions-One group received written invitations to have a smear taken. The second group had their notes tagged so that the doctor would remind them (when they attended for another reason) to have a smear test. No special intervention was made in the third group.

Main outcome measure-Performance of a cervical smear test during the year of the study.

Results - 32\% (45/140) Of the screened group, $27 \%$ (39/142) of the tagged group, and 15\% (20/134) of the control group had a smear test during the year. The percentage of women having a smear test in the screened group was not significantly different from that in the tagged group, but the percentages in the two groups were significantly different from that in the control group. Whether a woman had had a previous smear test significantly affected the uptake of the invitation to have a smear test independently of the method of invitation.

Conclusions - The systematic methods of call and recall were more effective than a non-systematic method. There was no significant difference between the two systematic methods (sending letters or tagging the notes) at one year.

\section{Introduction}

Evidence from other countries suggests that when organised cervical screening programmes are established and reach a high proportion of the women at risk mortality from cervical carcinoma is subsequently reduced. ${ }^{1-3}$ In England and Wales national cervical cytology screening services were established in 1964 but have not resulted in a reduction in the mortality from cervical cancer. ${ }^{4}$ This failure of the British cervical cytology screening programme has been attributed mainly to poor organisation resulting in failure to reach women most at risk, particularly those who are older or socially disadvantaged, or both. ${ }^{56}$ Suggestions to improve the cervical cytology screening programme have emphasised the importance of basing it around the general practitioner services. ${ }^{6}$

This raises the question of how general practitioners might most effectively approach women to ensure that cervical smear tests are carried out. Three different approaches might be suggested: $(a)$ a screening approach, all women at risk being sent a written invitation to have a smear test; $(b)$ a systematic opportunistic screening or case finding approach - the notes of eligible women are tagged, reminding the doctor to ask at any consultation about taking a smear; and $(c)$ an unsystematic opportunistic screening or case finding approach, which relies on the doctor approaching women about their smear tests during the course of their routine consultations without any reminder in the notes.

Much has been written about the theoretical advantages and disadvantages of each of these approaches, ${ }^{7-10}$ and the rates of uptake of an invitation to have a smear test with the individual approaches have been reported. ${ }^{11-14}$ The results of comparative studies have, however, not been reported. In this study we compared the effectiveness of the different approaches in one general practice.

\section{Methods}

The study was carried out in one group practice with a list of 10120 patients. The population consisted predominantly of people in the lower socioeconomic groups. All women born between 1926 and 1952 were identified from the age-sex register. From this group were excluded any women known to have had a hysterectomy and those who were on the practice's recall register for cervical cytology screening or who were otherwise known to have had a smear test within the previous five years. The women included were stratified according to age and randomly allocated to three groups: (a) the screening group (group 1); (b) the systematic opportunistic screening or case finding group (group 2); and (c) the control group, who received the usual unsystematic opportunistic screening or case finding.

Letters were written to the women in group 1 asking them to have a smear test. The notes of the women in group 2 were tagged with a partially completed request form for a cervical smear test that reminded the doctors to ask about cervical cytology screening at any consultation. No intervention was made in group 3. Group 3 had two functions: to act as a control group to see the effect of interventions unrelated to the study that might have arisen during the time of the studyfor example, educational campaigns on cervical cancer - and to show the effect of unsystematic opportunistic screening.

The doctors' surgeries were equipped to take smears and the two practice nurses (AP and SW) held a cervical smear clinic weekly as well as taking smears in their daily routine clinics. During the study the doctors and nurses were asked not to change their behaviour with respect to taking smears, except that when a woman's notes were tagged the doctor should advise the woman to have a smear test or to make an appointment to have one done at a later date. If a

Dr Pierce. 
woman attended for a smear test the person taking the smear was asked to find out why she had come to detect women attending for reasons other than the study interventions.

The data we collected initially were the date of birth, randomised group, and date of previous smear test (before study). One year after entry into the study the data collected were $(a)$ whether a smear had been done during the study and, if so, when and by whom; $(b)$ the reason for attending for the smear test during the study; $(c)$ the date last seen by the doctor; and $(d)$ the date of removal from the list because of having moved house or having died. The data were analysed with $\chi^{2}$ tests, confidence intervals, and a multiple log linear model.

\section{Results}

There were 1232 female patients born between 1 January 1925 and 1 January 1952 registered with the practice. Of these, 650 were already on the recall system and 166 had had a hysterectomy. This left a group of 416 women to be included in the study. Of these, 140 women were randomly allocated to the screening group (group 1), 142 to the tagging group (group 2), and 134 to the control group (group 3).

During the study 61 women were removed from the practice's list: 24 in the screened group, 20 in the tagged group, and 17 in the control group. Three women died and 58 left the practice. The number of deaths is accurate. All the migration figures were checked by contacting the patients' address to see whether they had moved. As a further check all women included in the study who had not been seen within the year of the study were contacted to see whether they were still registered with the practice.

In group 1 (the women receiving letters) most smear tests $(34 / 45(76 \%))$ were done within eight weeks of entry; in group 2 (women whose notes were tagged) and group 3 (the control group) the taking of smears was spread evenly through the year. This was as expected considering the interventions in each group.

The table shows how many women in each group had a smear test during the study. It shows that both screening and tagging were more likely than no intervention to result in women having a smear test. There was no significant difference in the proportion of women having smear tests in the screened group (group 1) compared with that in the tagged group (group 2).

The table also shows how the relation between the method of recall and attendance for a smear test during the study was affected by a woman having had a test more than five years before. These results show that women who had had a test before were more likely to attend for a smear test during the study independently of the recall system used.

Proportions (percentages) of women in each group who had smear test during study in total and according to whether they had had smear test more than five years before

\begin{tabular}{lccc}
\hline Randomised group & $\begin{array}{c}\text { Previous } \\
\text { smear test }\end{array}$ & $\begin{array}{c}\text { No previous } \\
\text { smear test }\end{array}$ & Total \\
\hline Screened & $24 / 52(46)$ & $21 / 88(24)$ & $45 / 140(32)^{\star} \dagger$ \\
Tagged & $20 / 51(39)$ & $19 / 91(21)$ & $39 / 142(27)^{\star} \dagger$ \\
Control & $9 / 37(24)$ & $11 / 97(11)$ & $20 / 134(15)^{\star}$ \\
\hline Total & $53 / 140(38) \ddagger$ & $51 / 276(18) \ddagger$ & $104 / 416(25)$
\end{tabular}

^Difference between percentage of women having smear test in screened and tagged groups and percentage in control group $=15 \%(95 \%$ confidence interval $7 \%$ to $23 \%)\left(\gamma^{2}=10 \cdot 70, \mathrm{df}=1, \mathrm{p}<0.01\right)$

tDifference between percentage of women having smear test in screened group and percentage in tagged group $=6 \%(95 \%$ confidence interval $-5 \%$ to $17 \%)\left(\chi^{2}=0 \cdot 74, \mathrm{df}=1,0 \cdot 3<\mathrm{p}<0 \cdot 4\right)$.

to $17 \%)\left(\chi^{2}=0.74, \mathrm{df}=1,0.3<\mathrm{p}<0.4\right)$. †Difference between percentage of women having smear test who had had a $(95 \%$ confidence interval $10 \%$ to $29 \%)\left(\chi^{2}=43.65, p<0.001\right)$.
No significant difference was found in any of the randomised groups in the proportion of women in each age group $(35-39,40-44,45-49,50-54,55-59$, and 60-62) who responded positively to an invitation. The numbers in each group were, however, small.

The factors that might influence the uptake of smear tests were examined simultaneously by using a $\log$ linear model. This confirmed that uptake was increased among those approached (groups 1 and 2) in comparison with the control group, that those with a previous smear test were much more likely to attend, and that the two effects were independent. Age group was not a significant factor when the other variables were allowed for.

Eighty per cent of the smears in all of the groups were taken by the practice nurses. Ten per cent were taken by the general practitioners and $10 \%$ by others (in hospital outpatient departments, screening services through employers, and well women clinics run by BUPA (British United Provident Association)).

\section{Discussion}

Our results show that both systematic methods of calling and recalling women for cervical cytology screening were more effective-that is, resulted in more women having a smear taken-than the unsystematic method. This agrees with Olesen's findings in Denmark. ${ }^{15}$ We, however, found no significant difference at the end of the year in the results of the two systematic methods of recall (groups 1 and 2).

More women were removed from the practice's list from group $1(24 / 140(14 \%))$ than from group $2(20 / 142$ $(8 \%)$ ) or group $3(17 / 134(6 \%))$. Most of the smear tests in group 1, however, were performed within eight weeks of entry. During this time only five of the 140 (4\%) women in group 1 were removed from the list.

In group 2 smears were taken evenly throughout the year. This, combined with the fact that $27 \%$ of the women in group 2 did not consult the doctor during the year of the study and so the doctor had no opportunity to invite them for a smear test, suggests that if the study continued with the notes left tagged the uptake in group 2 might have been higher than that in group 1 . Other studies have shown that $75 \%$ of patients see their general practitioner every year and that $90 \%$ of patients do see their general practitioner within five years. ${ }^{16}{ }^{17} \mathrm{It}$ might also be argued that as every woman in group 1 was sent a letter at the beginning of the study but only $73 \%$ of the women in group 2 were recommended smear tests by their general practitioner throughout the year the effective follow up time in group 2 was much less than that in group 1 .

Our aim, however, was to compare the effectiveness of the three different policies of recall available to a general practitioner. We did not intend to compare the delay from receiving an invitation to having a smear taken in women who received a letter and those who were approached by their general practitioner. Neither did we intend to compare the effectiveness of being sent a letter of invitation with being invited personally by the doctor, all other things being equal. Omission of women from group 2 who were never approached would, of course, increase the percentage who had a smear test during the study but would not fairly assess the policy. Inherent delay is also part of the policy being assessed. It was a pragmatic rather than an explanatory trial, as differentiated by Schwartz and Lellouch. ${ }^{18}$

Other work has shown that older women dying of carcinoma of the cervix are likely to have never had a cervical smear test ${ }^{19}$ and that older women are less likely to use the cervical cytology screening services. ${ }^{20}$ In each of the groups of women having systematic 
intervention in our study, however, the response rate was not affected by age, which confirms the findings of Ridsdale,$^{13}$ although the numbers in each of our groups were small.

Our findings suggest that if women were invited to have a smear test by a doctor they would accept the invitation irrespective of how old they are. This implies that the current failure of the British cervical cytology screening programme to screen women at high risk might be due partly to doctors failing to tell such women that they need to have a smear test rather than to women's reluctance to respond positively to such an invitation.

We also found that women who had had a smear test at any time were much more likely to respond positively to an invitation (by whatever means) than women who had never had a smear taken. Nationally more younger women are having smear tests. ${ }^{4}$ If the results of this study applied to other groups of women national coverage might be greater in future as younger women "get into the habit" of having smears taken.

We have five main conclusions from this study of a population of women over 35 with a predominantly low socioeconomic state who had not had a smear test within the previous five years. Firstly, systematic methods of call and recall are more effective than a non-systematic approach. Secondly, there are no differences in uptake at one year between a systematic case finding and a screening approach. Thirdly, there are no significant differences between randomised groups in response rate to invitation according to the age of the patient. Fourthly, women who have had a smear test in the past are more likely to attend for a test again. Fifthly, most smears were taken by the practice nurses.

We suggest that to improve the proportion of women having smears systematic simple intervention (written invitation or tagging notes) should be used and that much of the extra work can be undertaken by practice nurses.

1 Laara E, Day NI, Hakama M. Trends in mortality from cervical cancer in Nordic countries: associations with organised screening programmes. Lance 1987;i:1247-9.

2 Anderson GH, Boyes DA, Benedet JL, et al. Organisation and results of the cervical cytology programme in British Columbia, 1955-85. Br Med 1988:296:975-8

3 McGregor JE Teper S. Mortality from carcinoma of the cervix. Lance 1987:i:103()-2.

4 Roberts A. Cervical cytology in England and Wales 1965-80. Health Trends 1982;14:41-3.

5 Chamberlain J. Which prescriptive screening programmes are worthwhile? 7 Epidemiol Community Health 1984;38:270-7.

6 ICRF Coordinating Committee on Cervical Screening. Organisation of a programme for cervical cancer screening. Br Med f 1984;289:894-5.

7 Oliver MF. Strategies for preventing and screening for coronary heart disease. Br Hearl f 1985;54:1-5.

8 Sackett DL, Holland WW. Controversy in the detection of disease. Lancel 1975;ii:357.

9 Freer $\mathrm{CB}$. Geriatric screening: a reappraisal of preventative strategies in the care of the elderly. 7 R Coll Gen Pract 1986;35:288-90.

10 Fraser $\mathrm{R}$. The reliability and validity of the age/sex register as a population denominator in general practice. I $R$ Coll Gen Pract 1978;28:283-6.

11 Standing P. Mercer S. Quinquennial cervical smears: every woman's right and every general practitioner's responsibility. Br Med f 1984;289:883-6.

12 Briscoe $M$, Woods JO. Screening for cervical cancer in general practice. Ulster Med J 1984;53:76-9.

13 Ridsdale LL. Cervical screening in general practice: call and recall. $f R \mathrm{Coll}$ Gen Pract 1987;37:257-9.

14 Lang CC, Duncan ID, Duguid HLD. Opportunistic cervical screening in the Dundee catchment area. Health Bulletin 1985:43(4):156-65.

15 Olesen F. Use of pap smear in general practice by case finding or screening programme. Scand f Prim Health Care 1986;4:59-62

16 Hicks D. Primary care: a review. London: HMSO, 1976:161-7.

17 Secretaries of State for Social Services. Primary care: an agenda for discussion. London: HMSO, 1986

18 Schwartz D, Lellouch J. Explanatory and pragmatic attitudes in therapeutic trials. 7 Chronic Dis 1967;20:637-48

19 Ellman R, Chamberlain J. Improving the effectiveness of cervical cance screening. I R Coll Gen Pract 1984;34:537-42.

20 Savage W, McPherson A. Cervical cytology in women's problems in general practice. Oxford: Oxford University Press, 1983. (Oxford general practice series 4.)

(Accepted 14 fune' 1989)

\section{MATERIA PARAMEDICA}

\section{A tale of two holidays: how to make great discoveries}

When you go on holiday, do you down tools and push off? Do you leave equipment and materials on the bench lying round haphazardly, or do you tidy up to find everything neat on your return? There are two remarkable instances of the "down tools" method (if "method" can be appropriated for the unmethodical) that led to momentous discoveries in the treatment and prevention of infections.

In 1928 Alexander Fleming was investigating what factors influenced the colour changes in staphylococcal colonies. He found that leaving the petri dishes on the bench at room temperature was one of the relevant factors. So the cultures were left out and examined at weekly intervals. In late July Fleming left for his holiday home in Suffolk. He had shifted a number of dishes into a pile at the end of the bench, where they would be out of the sunlight and out of the way of his assistant, Stuart Craddock. Early in September Fleming examined and sorted out those cultures that might be of interest. Those he did not want he placed in a shallow tray containing lysol to kill all microorganisms before disposal. Some of the unwanted dishes, however, were high and dry above the level of the lysol. His erstwhile assistant, D M Pryce, looked in to see how things were going. Fleming showed him some of the discarded dishes and said, "That's funny." He had picked up the famous culture plate on which there was a large colony of the mould, penicillium, surrounded by a zone of disappearing staphylococcal colonies. ${ }^{1}$ I don't need to go on. Now, what would have happened, or rather "not happened," if Fleming had not left the culture plates lying around for four to five weeks, or if he had with greater care immersed all of the unwanted ones in lysol? Mind boggling, is it not?

In the 1870 s chicken cholera was epizootic in France and killing very many fowl. A microbe had been isolated from infected birds, and Louis Pasteur wanted to prove its causative role. He made a pure culture and found that it was highly virulent when injected into chickens. He then made a series of subcultures in chicken broth and found that they were all virulent. In the summer of 1879 Pasteur took a three months' holiday in
Arbois, his home town. He left the last subculture behind in the laboratory. On his return he injected the culture into chickens. Nothing. happened. He then prepared a fresh culture of known virulence. Again nothing happened to the chicks that had previously received the old culture. ${ }^{2}$ He had discovered attenuation of a virulent microorganism as a means of inducing immunity. As is well known, he went on from there to prepare successful attenuated vaccines against anthrax and rabies. Now just suppose Pasteur had started his experiments some months earlier or later and had consequently not gone on holiday; or suppose he had discarded the last subculture before leaving for Arbois, knowing that he would be away for three months. Perhaps he left in a hurry. I can almost hear Mme Pasteur pleading, "Come on, Louis; the carriage is waiting!"

And when Alexander and Louis came to publish their results, did either of them say, "I went on holiday," "I forgot to clear up the plates," or "I forgot to throw away the broth?" They did not. They had it all planned. Alex wrote: "cultures of 'staphylococcus variants ... were set aside on the laboratory bench and examined from time to time.",।

Louis wrote: “... by merely placing a longer interval of time between successive seminations, we have obtained a method for decreasing virulence progressively, and finally get at a vaccinal virus which gives rise to a mild disease, and preserves from the deadly disease."

The benefits of these two discoveries to mankind have been incalculable, for they were starting points for other antibiotics and other attenuated vaccines. An element of chance often enters the process of successful research, and the fact is rarely mentioned in the published results. But who would admit to carelessness bordering on negligence as a contributory factor?

Going on holiday? Have a good time! - BERNARD J FREEDMAN

1 Macfarlane G. Alexander Fleming: the man and the myth. London: Chatto and Windus/Hogarth Press, 1984:118-9.

2 Harré R. Louis Pasteur. In: Great scientific experiments. Oxford: Oxford University Press, 1983:100. 